\title{
Managing Initial Coin Offerings: Towards a Taxonomy of ICO Processes
}

\author{
Nikolaus Lipusch, Dominik Dellermann, Philipp Ebel \\ University of Kassel, Hessen, Germany \\ \{lipusch, dellermann, ph.ebel\}@uni-kassel.de
}

\begin{abstract}
Initial Coin Offerings are a new type of crowdbased fundraising mechanism that uses the blockchain to issue tokens to a crowd of people in exchange for funds that blockchain start-ups use to develop their business. Unfortunately, due to the recency of this new phenomenon, there is no systematic understanding of the ICO process and its underlying process characteristics. However, companies engaging in ICOs should be able to evaluate and choose the right process steps to best achieve their goal. Against this background, we develop a taxonomy for ICO processes. In contrast to previous work, this classification scheme focuses exclusively on the processual nature of ICOs and its underlying mechanisms.
\end{abstract}

\section{Introduction}

The blockchain receives a lot of attention in the financial and the information technology industry these days and is hailed by some proponents as the most disruptive technology since the web [1]. Generally, a blockchain is a distributed digital ledger that is characterized by five basic principles, namely a distributed network, peer-to-peer interaction, transparency with pseudonymity, irreversibility of the entries and programmability $[2,3]$.

Although the principles that make up the blockchain are not entirely new, their combination (i.e. the blockchain) is inextricably linked with increased innovation in various fields and application domains. The most prominent example is bitcoin, which provided the financial industry with a more efficient and reliable payment system. At the heart of bitcoin's blockchain is a so-called distributed ledger that allows not only to get rid of a middle-man, who governs and oversees all transactions, but also allows a more tamper-resistant system since transactions are recorded and validated by multiple users of a network. Newer generations of blockchain technology are even more disruptive in that they allow to represent a

\footnotetext{
${ }^{1}$ https://www.coindesk.com/257-million-filecoinbreaks-time-record-ico-funding/
}

variety of other business logics that go beyond financial transactions $[4,5]$. One example is Ethereum that can be used to represent a variety of functionalities such as virtual shares, assets, proof of membership and many others.

With the steady development of blockchain technology, also new use cases emerged. Initial Coin Offerings (ICOs) denote a new kind of fundraising method made available by the development of blockchain technology and cryptographic tokens. Start-ups can use this method to obtain crowd capital to fund and develop their blockchain projects. In exchange for capital these companies emit tokens through the blockchain that grant certain rights to investors. These rights can vary from project to project and entail things like access to a platform, application or service, rights to contribute work (e.g. developing or creating features for a system), rights to participate in a company's revenues, as well as rights to cast a vote on governance issues, etc. [6].

ICOs are currently experiencing a real boom. A prominent example is Filecoin, a US-based start-up that recently managed to raise $\$ 257$ million through an $\mathrm{ICO}^{1}$. In comparison, the highest amount of capital raised by a crowdfunding campaign (i.e. the Pebble smartwatch) was $\$ 20,3$ million. Despite the economic realities of this new phenomenon, research on ICOs is still in its infancy. Most research on ICOs is anecdotal and describes the greater phenomenon but leaves out detailed knowledge about ICO processes. However, when conducting an ICO a company must carefully consider between different decisions and actions that can be taken at each process step of an ICO. Unfortunately, current literature leaves entrepreneurs and start-ups in the dark, when it comes to figuring out, how they can use ICOs to achieve their goals and which process steps they need to follow to reach them. Against this background, this paper tries to answer the following research question:

What processes and process characteristics must a blockchain start-up consider during an ICO and how are these processes related to the goals a start-up is trying to achieve? 
The purpose of this paper is to propose a systematic scheme (i.e. a taxonomy) for classifying ICO processes. To this end, we analyze data of a representative sample of 42 ICO campaigns as well as literature related to the phenomenon. By developing a taxonomy of ICO processes, we aim to contribute to a better theoretical understanding of this rather young research domain. Additionally, we provide entrepreneurs with a guideline (in the form of a taxonomic framework) that they can use to strategically decide 1.) if an ICO is suited to achieve their goals 2.) and if so which process steps they must follow to achieve a certain goal.

The remainder of this paper is structured as follows: In section two we cover the related work and the conceptual background of ICOs. In section three we provide a general explanation of our methodological approach and how we applied it to derive our taxonomy. In section four we present the results of our research (i.e. the taxonomy as well as the identified clusters). We summarize the major findings in section five. Finally, we elaborate on possible limitations and future research in section six.

\section{Related Work and Conceptual Background}

Before we introduce ICOs, we provide an overview of related work and important concepts such as the blockchain, smart contracts, cryptocurrencies and tokens and crowdfunding.

\subsection{Blockchain and Smart Contracts}

The blockchain was first introduced in Satoshi Nakamoto's paper on a decentralized payment system called bitcoin in 2008 [7]. At that time the blockchain was described as a decentralized shared ledger that uses chronological, encrypted and chained blocks to store verifiable and synchronized data across a peerto-peer (P2P) network [8]. By using the blockchain, bitcoin was able to bypass intermediaries through socalled miners (i.e. the P2P network) who contribute their computing power to verify transactions that are summarized in blocks and then stored in a shared ledger (i.e. the blockchain) [9]. With advances in the blockchain technology (i.e. blockchain 2.0), the functionality of the blockchain increased vastly. Thus, the second generation of blockchains moved beyond Bitcoin's single purpose of transferring cryptocurrencies. One example of such a blockchain is Ethereum that due to its Turing-complete programming language offers a generally programmable platform that can be used as infrastructure for a variety of applications [10]. Thus, Ethereum can be used for purposes such as controlling digital assets, identity management and fundraising
[10]. Another important feature of these newer generations of blockchains are so called smart contracts. Smart contracts refer to programs that are executed on the blockchain and that can be used to automate any of the business logics and applications mentioned before $[11,12,5]$.

\subsection{Cryptocurrencies and Tokens}

One term that is inextricably linked with the blockchain are so called cryptocurrencies. The most popular example is again Bitcoin. At the heart of bitcoin are so called (bit)coins that denote a digital payment system. Coins can thereby be used as a medium to store and transfer value within a network [7]. The main advantage of such a decentralized payment system is that users are not dependent on intermediaries to handle their transactions, meaning that users have greater freedom to engage in borderless and frictionless transactions [9]. With the second generation of blockchains (i.e. Ethereum), tokens became more popular. Although coins and tokens are often used synonymously there is a fundamental difference between those two concepts. According to the Cambridge dictionary, tokens denote "a round, metal or plastic disk which is used instead of money in some machines". Hence, tokens can be best understood as a voucher or a gift card that can be used to consume a variety of services within a certain context (e.g. a shop, a fair, a casino or a vending machine). This is different from coins and cryptocurrencies, which usually act as a medium to transfer value across a variety of contexts. Another distinguishing characteristic of tokens is that they are programmable. One consequence of this is that they can be programmed to serve a variety of different functionalities and purposes. For example, they can be used to facilitate transactions, as an internal unit of account, for the verification of block-writing, or for more creative uses such as helping to prevent unintended use of the blockchain and to grant token holders certain types of privileged access [6, 5, 13]. It is important to note that these are just some examples and that some tokens can fulfill one or several of the above-mentioned functions.

Apart from that it can be distinguished between native tokens inherent to a blockchain - so called protocol tokens - and on-chain tokens (sometimes referred to as app coins or app tokens) that are issued on top of a blockchain using smart contracts [10, 14, 15]. While native tokens mainly serve as incentive to develop and operate the blockchain, app-coins are tokens that can be used to access specific applications (i.e. the services) that are built on top of the blockchain. The most popular standard used to create 
app coins is the ERC20 standard that is employed by the Ethereum blockchain [16].

As diverse as token functionality is, as diverse are their use cases. For example, tokens can act as an access key that developers can use to contribute work (i.e. work tokens). Another example constitutes tokens that act like shares (i.e. equity tokens) that allow developers to participate in the potential rise of value of the ecosystem they are building. Furthermore, as mentioned before native tokens inherent in the blockchain are used to incentivize miners to maintain and operate the network. This is achieved through so called proof-of-work algorithms that reward miners for solving cryptographic puzzles on the blockchain [9]. Lastly, tokens can be issued in the form of ICOs in exchange for payment. These ICOs are regularly used by start-ups to collect the necessary funds to develop their blockchain projects [6].

\subsection{Crowdfunding}

Crowdfunding is defined as "a collective effort by people who network and pool their money together, usually via the internet, in order to invest in and support efforts initiated by other people or organizations." [17]. The main rationale behind this concept is to collect small funding increments from a crowd of investors, which add up to a significant investment that start-ups can use to develop their business. In recent years, crowdfunding developed as a serious fundraising alternative for start-ups that are not eligible to traditional means of financing such as bank loans and venture capital. One popular example constitutes the Pebble smartwatch which raised 20.3 million US\$ in funding. Despite its recent success, crowdfunding is also characterized by certain weaknesses. Thus, users of crowdfunding are usually charged a commission fee based on the total funds raised [18]. Further costs arise due to auxiliary services such as payment providers, which are necessary to process payments among the involved parties [19]. Another issue concerns the trust between capital givers and capital seekers. At the heart of this are information asymmetries between capital seekers and capital givers which usually put capital givers at a higher risk due to holding incomplete information. While crowdfunding platforms formed as a solution to mitigate these problems, the mechanisms used by these platforms sometimes perform very weakly (see [20-23]. Also, the mechanisms employed by crowdfunding platforms constrain how crowdfunding can be conducted [18]. ICOs developed as a new crowdfunding mechanism that bears the potential to solve these problems [13]. In the following, we introduce the concept of ICOs and elaborate how it differs from previous approaches to crowdfunding.

\subsection{ICOs: A New Type of Blockchain-based Crowdfunding}

ICOs, also often referred to as "token-sales" or "crowd-sales", recently emerged as a new business model that allows blockchain start-ups to collect capital to realize their business (usually before the business is initiated). Blockchain start-ups refer to businesses in the blockchain domain which main aim is to develop blockchain protocols as well as blockchain applications [6]. Since ICOs share a lot of similarities with crowdfunding (e.g. they are conducted over the web and rely on the principle of crowdsourcing) they are considered as a new crowdfunding mechanism $[13,18]$. However, one important difference to conventional crowdfunding mechanisms is that ICOs are conducted via a blockchain. The main advantage of this is that ICOs function in a completely decentralized way through peer-to-peer mechanisms and, hence, do not require a central intermediary that moderates the matchmaking process between project initiators and investors [24, $19,13]$. While this allows ICOs to be cheaper, this is also likely to alter the processual nature of ICOs compared to crowdfunding.

Figure 1 illustrates a prototypical ICO process. A start-up engaging in an ICO uses the blockchain to generate tokens that will be issued to potential investors. Hence, the blockchain constitutes the technological infrastructure upon which a company creates and issues tokens. As we have already mentioned before, such tokens can represent different utilities (see 2.2). In most cases they represent an access right to consume the services that are provided by the start-up (also via the blockchain). In exchange for tokens the company receives investments from a crowd in the form of cryptocurrencies (most often Bitcoin or Ethereum). The individual investments of the crowd are then pooled together to finance the development of the blockchain project (e.g. to cover the costs of developers).

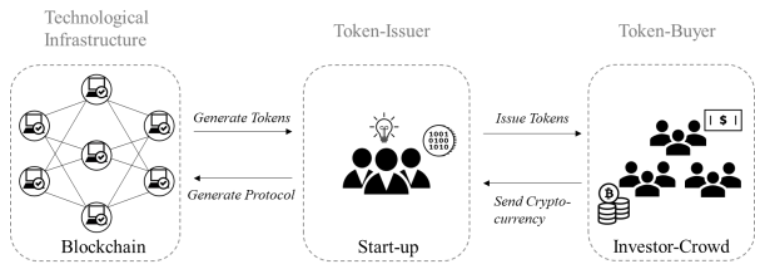

Figure 1. Prototypical ICO Process

As can be seen from our illustration above, ICOs differ significantly in their structures and processes from related fundraising mechanisms. To get a better and more detailed understanding of these processes, research is needed. 


\section{Methodology}

In the following section we provide a general overview of what constitutes a taxonomy. Furthermore, we explain in detail how we derived our taxonomy.

\subsection{Taxonomical Approach}

Taxonomies play an important role in structuring and ordering new concepts and hence lay the foundation to postulate and hypothesize about relationships among these concepts $[25,26]$. To derive our taxonomy, we rely on a method proposed by Nickerson et al. [27] who came up with a design-based approach for taxonomy development [28], that allows to identify the dimensions (or variables) and corresponding characteristics (or variable domains) of the taxonomy through an iterative design process. By applying this approach, we follow seven general steps (see Figure 2).

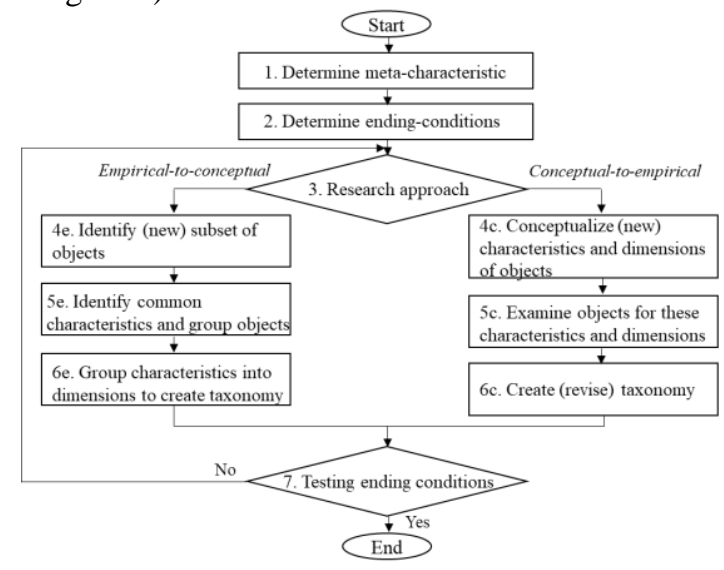

Figure 2. Taxonomical approach (source: Nickerson et al. 2013)

\subsection{Research Approach to Derive Our Taxonomy}

Following the approach proposed by Nickerson et al. (2013) [27], we define our meta-characteristic in step one. This is the most important step as it helps to determine the purpose of the taxonomy with a view to its main target group. Since the intended users of our taxonomy are new ventures that possess limited knowledge with regard to the purpose and functioning of ICOs, we frame our meta-characteristic as follows: We develop a taxonomy for design parameters and characteristics of ICO processes that blockchain startups can use to decide on how to conduct ICOs in a way that best serves their goals.

\footnotetext{
${ }^{2}$ This was to increase the reliability of our results.
}

In a second step, we determine our ending conditions. For our ending conditions we made use of objective as well as subjective ending conditions (see [27]). In regard to our objective ending condition, our taxonomy must consist of dimensions with mutually exclusive and collectively exhaustive characteristics. For the subjective ending conditions, we decided to apply those proposed by Nickerson et al. (2013), who note that a useful taxonomy must be concise, robust, comprehensive, extendible and explanatory (for a detailed explanation see [27]).

In step three we chose our research approach. As proposed by Nickerson et al (2013), we employ an empirical-to-conceptual- as well as a conceptual-toempirical -approach [27].

For the empirical-to-conceptual approach we decided to collect and analyze 42 real life ICOs (this corresponds to step 4e) from the years of 2014 to 2018. To collect our sample of ICO campaigns, we made use of websites such as CoinSchedule, TokenMarket and Coinbase which contain an overview of historic, ongoing and upcoming ICOs. To analyze the ICOs, we primarily relied on secondary data and made use of multiple data sources ${ }^{2}$ (see Table 1). The gathered data was used to identify common process characteristics and design parameters of ICOs (step 5e). To extract meaningful process characteristics, we made sure to only include characteristics that discriminate among the analyzed ICOs in a sufficient manner (see [27, 29]). In a next step (step 6e), we used a manual procedure to group the identified characteristics into dimensions (i.e. higher order concepts). This resulted in five dimensions (see Figure 3), which can be best understood as more abstract processes that contain the mutually exclusive process characteristics that we identified earlier.

\begin{tabular}{|c|c|}
\hline Data Sources & Examples \\
\hline $\begin{array}{l}\text { - Press releases, news, } \\
\text { announcements, online } \\
\text { articles }\end{array}$ & $\begin{array}{l}\quad \text { e.g., CoinDesk, } \\
\text { BraveNewCoin, } \\
\text { CoinTelegraph, Medium } \\
\text { etc. }\end{array}$ \\
\hline $\begin{array}{l}\text { - Case Documents, Legal } \\
\text { Papers and Technical } \\
\text { Papers }\end{array}$ & $\begin{array}{l}\text { e.g., Whitepapers, } \\
\text { Yellow papers, Legal-term } \\
\text { sheets etc. }\end{array}$ \\
\hline - Keynotes and Speeches & $\begin{array}{cr}\text { e.g., } & \text { DevCons, } \\
\text { Deconomy, } & \text { Blockchain } \\
\text { labs, Slide decks etc. }\end{array}$ \\
\hline Websites, Platform data & $\begin{array}{c}\text { e.g. Company } \\
\text { websites, Company blogs, } \\
\text { Company newsletters etc. }\end{array}$ \\
\hline
\end{tabular}




\begin{tabular}{|c|c|}
\hline $\begin{array}{l}\text { - Social Media Data and } \\
\text { Databases }\end{array}$ & $\begin{array}{l}\text { e.g. Reddit, Telegram, } \\
\text { GitHub, StackExchange } \\
\text { and Gitter conversations } \\
\text { etc. }\end{array}$ \\
\hline
\end{tabular}

Table 1. Secondary data sources used for creating our taxonomy

For our empirical-to-conceptual approach we analyzed literature related to the identified process characteristics and dimensions. In doing so, we utilized literature on crowdfunding, IPOs, venture capital and auction mechanisms. This additional step allowed us to verify our existing processes as well as to conceptualize new process characteristics for our taxonomy (i.e. step 4c). Finally, we took a sub-sample of our ICOs to verify the applicability of the newly derived process characteristics (i.e. step 4c) and adapted our taxonomy accordingly (i.e. step 5c). Steps 3-7 were repeated several times, checking against our ending conditions in each iteration, until we arrived at our final taxonomy

To determine the usefulness of our taxonomy, we performed an additional evaluation cycle. In doing so we conducted interviews with three experts that had either acquired relevant practical knowledge or made a significant scientific contribution in the field of ICOs. The feedback of the experts indicates that our taxonomy could be useful for "start-ups who are interested in conducting ICOs especially against the background that best practices on how ICOs are conducted change very rapidly due to the very young nature of the phenomenon". Moreover, one of the experts noted that "Since ICOs are less formalized (e.g. they are not guided by intermediaries such as for example crowdfunding) and because they are technologically more complex than comparable fundraising options adequate guidelines in the form of such a taxonomy are very important to support entrepreneurial decision making during ICOs". Another expert noted that "a lot of companies naively rush into ICOs without considering if ICOs are the right type of financing for their business model. Providing a taxonomy could help companies to better assess if an ICO is the right way for them to develop their business".

\section{Findings}

In the following section we elaborate on the main results of our taxonomy creation process.

\section{1 Characteristics of ICO processes}

Throughout this research, we identified five dimensions that describe how ICO processes differ. Figure 3 depicts these dimensions and their logical order within an ICO process. First, the company considering an ICO must decide how it defines and approaches its market. Then, the new venture needs to decide on the functionality of the tokens i.e. which types of token it wants to create and issue. This is an important step as it defines what the tokens can be used for (i.e. the value proposition for the user) and how they interact with a company's business model. In a next step, the company needs to decide how it wants to create the tokens that are issued to the crowd in exchange for cryptocurrencies. When the organization has decided for a certain token creation strategy, it must determine the token sale model which determines how tokens are distributed. Finally, a company must decide on its user communication and engagement strategy. This is an important step to convince users of the feasibility and utility of the project as well as to engage them throughout the entire ICO. It is important to note here that while the proposed sequence follows a logical order, this might not reflect the actual order of process steps companies follow when conducting an ICO.

\subsubsection{Defining the Market}

The first dimension, defining the market, is concerned with determining the groups that are targeted by a company's ICO. Once this process is applied, selected crowd investors can decide if they want to contribute to the ICO or not. As part of our data analysis, we identified four process characteristics that are used to define the market: $a$ public offering, a public-offering with a pre-sale, a private offering and self-selection.

Some of the ICO campaigns we analyzed deployed a public offering. Public offerings are characterized by a maximum of openness, meaning that they do not limit the participation of buyers. One of the advantages of this process characteristic is that it allows companies to leverage a high number of users (i.a. also potential investors) which benefits the scalability of a project. Very often (but not always) public offerings came with a so-called pre-sale (i.e. a public offering with pre-sale). Pre-sales allow a company to issue a certain number of tokens beforehand (i.e. before most of the tokens are issued to the broader market). The benefit of such pre-sales is that they can help companies to better estimate the market potential for a company's tokens. Apart from that, pre-sales combined with a discount on tokens also constitute a promising strategy to attract early adopters.

Other ICO campaigns employed so-called private offerings. Private offerings differ from public offerings in that they are geared toward a specific group. Private offerings are often used to gather a core team around the project. Hence, this type of offering is often (but not exclusively) restricted to company owners, developer's advisors (e.g. advisor sales) and 
other important partners that take a key role in the creation of the project. This practice may be best compared to stock options that are offered to employees of a company.

Finally, some campaigns employed self-selection procedures which can be considered a mixture of the two characteristics mentioned above. Self-selection procedures require interested investors to register first on so-called whitelists to get considered for an offering. Some companies use this mechanism to determine market interest and to get order and fairness into the offering process by applying a first-come firstserve principle. Other companies use it as a selectionmechanism to weed out unsuitable investors (e.g. companies that are looking exclusively for accredited investors). Moreover, certain companies use this procedure to get customer information that is needed in certain jurisdictions to address "Know your Customer" and "Anti Money Laundering" regularities.

\subsubsection{Determining the Token Functionality}

The second dimension, determining the token functionality, is concerned with stipulating the purpose of tokens as well as choosing the right token standards to realize these purposes. It can be distinguished between five process characteristics: utility-based tokens, equity-based tokens, work-based tokens and asset-based tokens.

Utility-based tokens denote a process characteristic in which a company creates and issues tokens (socalled usage tokens) that permit token holders to use a certain product or service. This type of token can be best compared to pre-selling agreements that promise users access to digital services that are about to be developed and provided by the company conducting the ICO. These services can take on many different forms. For example, Filecoin tokens provide users access to decentralized storage.

Equity-based tokens, sometimes also referred to as tokenized securities, describe a process characteristic in which a company creates and issues tokens that represent a tradable financial asset. These types of tokens can be best compared to a digital share in a company that entitles token holders to equity-like benefits such as profit-sharing or voting rights.

Work-based tokens describe a process characteristic in which a company issues so-called work tokens in exchange for capital (i.e. cryptocurrency). Work tokens enable holders to contribute work to a network and earn value in exchange for their work [10].

Finally, asset-based tokens denote a process characteristic in which companies create and issue tokens that represent a physical asset. These tokens are useful as they allow for the digitization of physical assets and commodities. One example of a company using this type of token is Goldmint, which uses the blockchain technology to tokenize gold. The main advantage of such tokens is that they allow to manage the associated assets more efficiently (e.g. tokenized gold can be transferred and stored at lower costs).

\subsubsection{Token Development and Creation}

The third dimension, token development and creation, is concerned with the development strategy a company employs to create a token during an ICO. It can be distinguished between three process characteristics, namely native development, on-chain development and side-chain development.

The process characteristic native development means that the token to be developed is native (i.e. the token is inherent to a blockchain). Companies deploying this kind of process usually build a token from scratch. This means that the company has to create the token as well as the token's underlying infrastructure (i.e. a blockchain). While creating a token from scratch is associated with a lot of development effort, one of the main advantages of this process is that it provides companies with more flexibility in determining the token's functionality.

On-chain development denotes a process characteristic in which a company makes use of an existing infrastructure to create and develop its token (e.g. app tokens). This means that the token is developed on top of an existing blockchain. One of the most popular examples is the Ethereum blockchain which features its own token building standard (also known as ERC20) that allows to create tokens more easily through smart contracts. While developing a token on top of an existing infrastructure does not grant as much flexibility as native development, it significantly eases the process as it requires significantly less development effort.

The third process characteristic, side-chain development, is closely related to native development as it entails the creation of a so-called side-chain. Sidechains denote an additional blockchain aside a main blockchain. Side-chains are usually interoperable which means that tokens from one blockchain (e.g. the main chain) can be used on the other chain (i.e. the side-chain) and vice versa. Side-chains are usually employed by start-ups that want to test new tokens or new token models without compromising the functionality and security of the main blockchain.

\subsubsection{Determining the Token Sales Model}

The fourth dimension, determining the token sales model, describes the mechanisms by which a company aims to sell and distribute its tokens. During our empirical analysis, we identified four distinct process 
characteristics employed during an ICO: cappedsales, uncapped-sales, auction-sales and others.

The process characteristic, capped-sale, describes a restriction on the number of tokens that are issued during a token sale. This means that companies cap the amount of capital to be raised through an ICO by fixating the total token supply. Once this predetermined token supply has been consumed, the sale stops and there is no possibility for investors to obtain further tokens. During uncapped-sales, tokens can be usually availed at a first-come-first-serve basis at a fixed price. Moreover, a fixed or predetermined percentage of the total token supply is allocated to the core developers and founders.

Uncapped-sales denote a process characteristic in which a company sells an unlimited number of tokens at a fixed price over an extended period of time [30]. This means that investors can buy as many tokens as they desire. Due to their special characteristics, uncapped-sales are especially suitable for companies considering multiple investment rounds. Hence, the main purpose of uncapped-sales is to maximize both the number of investors involved and the amount of capital flowing into the project. Similar, to cappedsales, a fixed percentage of the total token supply is allocated to the founders and the development team.

Some of the ICOs we analyzed employed anauction-sale. This process characteristic denotes a special kind of sale in which buyers determine the price and the total amount they are willing to spend [31]. The issuing company then sells a variable number of tokens at the lowest bid price and in proportion to the total amount pledged. This type of mechanism is often used when a quick sale of tokens is desired. One example of an ICO that employed an auction sale was the Gnosis project with the aim to alleviate investors' fear of missing out. Participants in this sale are allocated a variable percentage of the total token supply, depending on the total number of tokens sold during the sale.

Quite recently, there has been an upsurge of new token-sales models. For our taxonomy we summarize them as others. These are sales that either constitute a mix of the three main sales models mentioned above or sales that cannot be subsumed under one of these models. Examples are dynamic-ceilings and soft-caps. A dynamic ceiling is considered as a series of mini hidden hard-caps set at specific block intervals. A softcap on the other hand refers to an extended time-based closing period until the full closure of the sale.

\subsubsection{User Communication and Engagement}

The fifth dimension, user communication and engagement, indicates how new ventures communicate and engage with their investors during an ICO. The dimension represents the four characteristics inform, consult, involve, and mixed, which reflect the degree of interaction between project creators (i.e. start-ups) and crowd investors.

The process characteristic inform denotes the lowest level of interaction and concerns the creation and provision of basic informational resources by the company. Most companies employing this type of process characteristic employ a website, a video, a whitepaper (i.e. basically a business plan of the blockchain project) or a yellow paper (i.e. a technical paper). While investors can use this information to get a basic idea about the project, it is important to note that this type of communication is non-interactive and non-binding. Hence, entrepreneurs can make no legal claims based on this information, nor do they have the possibility to inquire additional information they might be interested in.

Consulting goes beyond simple information provision. Usually this process characteristic involves one party inquiring or providing information that goes beyond the basic information requirements discussed above. Typical examples include surveys or questionnaires that companies use to determine the market needs of their customers. Other examples include terms of sale documents and purchase agreements that companies use to inform investors about their rights and risks [32]. While these documents are not legal documents in a strict sense they may be legally binding to a certain extent.

The process characteristic involve constitutes the highest level of interaction. It is characterized through multilateral and ongoing interaction between the company and the crowd investors. The main goal of this process characteristic is to establish the trust that is necessary to attract a community of loyal users. Popular channels that are used for this purpose are Reddit, Slack, Gitter or GitHub. Once a company manages to build and maintain a community, users of this community can be engaged in various activities that create value for the company. For example, they can be leveraged to contribute code via GitHub. Other examples include so called bounty programs, in which users contribute through identifying bugs in the software or promoting the project (either through word of mouth or through writing blog articles).

Finally, some ICOs employ a mix of the abovementioned process characteristics (e.g. inform and consult and engage) to communicate and engage with the crowd. For instance, Steemit, which operates a decentralized social network, features a website that features multiple versions of whitepapers and yellow papers. Additionally, Steemit communicates through several social media channels (e.g. Reddit and Slack) and organizes regular bounty programs (e.g. the midex 
bounty program and the deep onion bounty program) in which users are asked to promote the apps that are built on the Steemit network.

\subsection{Types of ICO processes}

Our proposed taxonomy contains five distinct dimensions that contain 19 process characteristics. By classifying the processes of our 42 ICOs we obtain a list of 33 distinct process types (i.e. combinational paths of process characteristics). In order to identify more generic archetypes among these process types, we additionally performed a cluster analysis [33, 34]. By doing so, we used a log-likelihood distance measure as well as Schwarz's Bayesian cluster criterion.

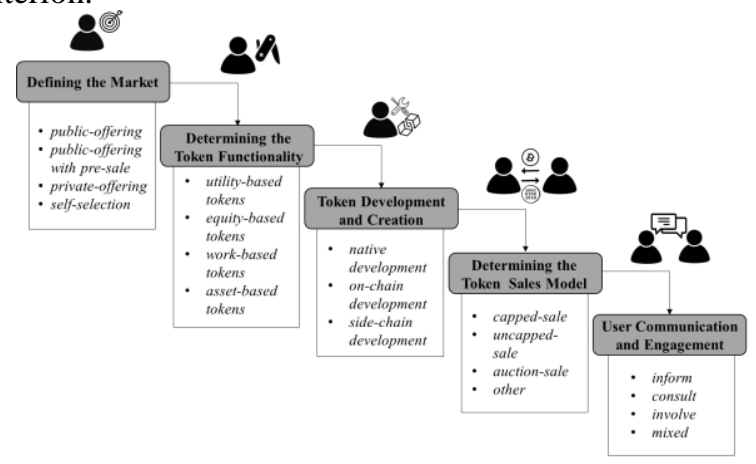

Figure 3. Dimensions and characteristics of ICO processes

Our analysis resulted in three robust clusters. In the following we provide a short description of each of the identified clusters. Cluster 1 - Customer-centric Service Innovators subsumes the biggest group of ICOs with $45 \%$. The cluster is mainly characterized by companies which aim to disrupt existing industries through new innovative business models and more customer-centric services. Hence, these types of companies very often employ utility-based tokens (68\%), which allows them to pre-sell access to their services to potential customers. Furthermore, this cluster also contains a decent number of asset-based tokens $(29 \%)$ that sells future assets to investors to be used within these new innovative business models (e.g. IoT). To define the market, ICOs in this cluster mainly employ public offerings as well as public offerings with pre-sales. One possible reason for this might be to reach as many customers as possible as well as to reach a sufficient amount of people to scale their business models. Regarding user communication and involvement, this cluster is characterized by intermediate to high interaction. This means that beyond using websites, whitepapers and blogs, a decent number of companies within these ICOs also use channels such as Reddit, Slack for purposes of determining customer needs. Most companies within this cluster develop their token on-chain (82\%) (e.g. on Waves or Ethereum). The most used token-sales model within this cluster of ICOs constitute cappedsales followed by uncapped-sales and auction-sales.

Cluster 2 - Financial Service Innovators subsumes the second biggest group of ICOs with $37 \%$. This cluster is mainly characterized by companies that are looking for capital and are mainly interested in selling financial products (hence most of these companies (74\%) employ equity-based tokens). Most often, these types of companies employ selective offerings (i.e. supposedly, to adhere to KYC and AML regulations) or public offerings (supposedly, to leverage greater amounts of capital). The interaction with crowd investors can be characterized as low to intermediate with most companies within this cluster employing websites, whitepapers as well as well as purchase agreements and legal sale documents. Moreover, most companies within this cluster develop their projects on chain (77\%), as compared to $14,5 \%$ of companies which develop their project natively and $8,5 \%$ of companies which develop their projects on a side- chain. The token sale models most often employed within this cluster, constitute capped-sales and auction-sales. One possible reason for this might be to create artificial scarcity among tokens to lure in investors.

Custer 3 - Platform Innovators contains the third biggest group of ICOs with $18 \%$. The cluster is mainly characterized by companies which aim to build and scale an ecosystem. Companies employing this type of ICO very often employ work tokens (33\%). One of the main reasons for this might be to pay the developers that build the ecosystem. Additionally, ICOs within this cluster also employ equity-based tokens $(66 \%)$. One reason for this might be to offer essential stakeholders (i.e. all parties that are necessary for the functioning of ecosystem) an additional incentive to participate. In regard to the definition of the market, a lot of ICOs within this group make use of private offerings as well as public offerings. Private offerings are thereby mainly used to attract a core team of developers that is needed to create the ecosystem. The public offering, on the other hand, is used to get the critical user traction that is needed to scale the network of the ecosystem. When compared to the other clusters, the user communication and involvement is characterized through high interaction. Thus, a high percentage $(81 \%)$ of ICO campaigns within this cluster employ all three communication strategies mentioned in 4.1 .2 (i.e. mixed). Also, the majority of ICO in this group makes use of so-called native tokens, meaning that they develop their own blockchain and its respective tokens. The most used token sale models within this cluster are uncapped-sales and others. One 
possible reason for this might be the high capital requirements that are needed to build an ecosystem (calling for no-cap sales models) as well as the complex ecosystem relationships and interactions that require more complex token sale models (calling for other sales models).

\section{Conclusion}

The goal of this research paper was to develop a taxonomy of ICO processes. Through our empirical analysis, we were able to categorize five distinct process dimensions and 19 process characteristics that make up our taxonomy. Furthermore, we identified three distinct ICO archetypes that can be used to fully describe our sample campaigns. Our results confirm and extend existing knowledge on ICOs. Thus, in line with the recently published taxonomy by [35] and the working paper of [32] we are able to show that ICOs differ along dimensions such as information disclosure, user engagement, sales terms and processes, token development and implementation, as well as registration processes. However, one important difference of our taxonomy compared to the taxonomy of [35] is that it follows a process-oriented logic. By doing so our taxonomy does not only provide insight with regard to what ICOs are on a theoretical level, but it also provides new ventures and entrepreneurs with prescriptive knowledge which may help them to assess which process characteristics to consider and which process steps to follow when conducting an ICO to achieve a certain goal. The goals of new ventures thereby correspond to different types (i.e. clusters) of ICOs identified in this research (i.e. the creation of customer centric service innovations, the creation of financial service innovations and the creation of platform innovations). Although the derived clusters differ from the ones identified by [35], they are easy to interpret and, thereby, likely to foster an intuitive understanding of ICO processes among entrepreneurs.

\section{Limitations and Future Research}

In accordance with Nickerson's approach to taxonomy building, our main aim was to build a useful taxonomy. While our first evaluation shows that our taxonomy is indeed perceived as useful, we are aware of the fact that the de-facto usefulness of our taxonomy can only be determined over time, through continuous and recurrent use of our artifact [28]. Another point to consider is that ICOs are still a very young phenomenon. Hence, knowledge on ICOs is still in a state of limbo with the potential to change or becoming obsolete very fast. One reason for this is that a regulatory on ICOs is yet to form and best practices on conducting ICOs change daily. Against this background, we like to point out that our taxonomy should be considered "as work in progress" Future research should, thus, focus on empirically validating our taxonomy as well as extending and adapting our taxonomy in line with regulatory changes that might occur. Moreover, our taxonomy might also constitute a promising starting point for empirical studies to examine how different process characteristics influence the success of ICOs.

\section{References}

[1] Beck, R. and C. Müller-Bloch, "Blockchain as radical innovation: a framework for engaging with distributed ledgers as incumbent organization", Proceedings of the 50th Hawaii International Conference on System Sciences, 2017, pp. 53905399.

[2] Iansiti, M. and K.R. Lakhani, "The truth about blockchain", Harvard Business Review, 95(1), 2017, pp. 118-127.

[3] Murck, P., "Who Controls the Blockchain", Harvard Business Review, 2017.

[4] Lindman, J., V.K. Tuunainen, and M. Rossi, "Opportunities and risks of Blockchain Technologies-a research agenda", Proceedings of the 50th Hawaii International Conference on System Sciences, 2017, pp. 1533-1542.

[5] Glaser, F. and L. Bezzenberger, "Beyond cryptocurrencies-a taxonomy of decentralized consensus systems", Social Science Research Network (SRRN), 2015, pp. 1-18.

[6] Conley, J.P., Blockchain and the Economics of Crypto-tokens and Initial Coin Offerings, Vanderbilt University Department of Economics, 1/1/2017.

[7] Nakamoto, S., "Bitcoin: A peer-to-peer electronic cash system", 2008.

[8] Yuan, Y., Wang, F. Y., "Towards blockchainbased intelligent transportation systems", in International Conference on Intelligent Transportation Systems (ITSC). 2016. IEEE.

[9] Prpić, J., "Unpacking Blockchains", arXiv preprint arXiv:1703.06117, 2017.

[10] Buterin, V., "A next-generation smart contract and decentralized application platform", white paper, 2014.

[11] Beck, R., M. Avital, M. Rossi, and J.B. Thatcher, "Blockchain Technology in Business and Information Systems Research", Business \& 
Information Systems Engineering, 59(6), 2017, pp. 381-384.

[12] Sillaber, C. and B. Waltl, "Life Cycle of Smart Contracts in Blockchain Ecosystems", Datenschutz und Datensicherheit-DuD, 41(8), 2017, pp. 497-500.

[13] Schweizer, A., V. Schlatt, N. Urbach, and G. Fridgen, "Unchaining Social Businesses-Blockchain as the Basic Technology of a Crowdlending Platform", Thirty Eighth International Conference on Information Systems, South Korea, 2017, pp. 1-21.

[14] https://github.com/DavidJohnstonCEO/Decentralized Applications, accessed 6-1-2018.

[15] Kuo Chuen, D.L., "Fintech Tsunami: Blockchain as the Driver of the Fourth Industrial Revolution.", Social Science Research Network (SRRN), 2017, pp. 1-11.

[16] https://github.com/ethereum/EIPs/issues/20, accessed 5-1-2018.

[17] Ordanini, A., L. Miceli, M. Pizzetti, and A. Parasuraman, "Crowd-funding: transforming customers into investors through innovative service platforms", Journal of service management, 22(4), 2011, pp. 443-470.

[18] Arnold, L., M. Brennecke, P. Camus, G. Fridgen, T. Guggenberger, S. Radszuwill, and A. Rieger, "Blockchain and Initial Coin Offerings: Blockchain's Implications for Crowdfunding", 2018, pp. 1-29.

[19] Haas, P., I. Blohm, and J.M. Leimeister, "An empirical taxonomy of crowdfunding intermediaries", in International Conference on Information Systems, Auckland, New Zealand. 2014.

[20] Lehner, O.M., E. Grabmann, and C. Ennsgraber, "Entrepreneurial implications of crowdfunding as alternative funding source for innovations", Venture Capital, 17(1-2), 2015, pp. 171-189.

[21] Mollick, E., "The dynamics of crowdfunding: An exploratory study", Journal of business venturing, 29(1), 2014, pp. 1-16.

[22] Mollick, E., "The dynamics of crowdfunding: An exploratory study", Journal of business venturing, 29(1), 2014, pp. 1-16.

[23] Lipusch, N., D. Dellermann, S. Oeste-Reiß, and P. Ebel, "Innovating Beyond the Fuzzy Front End: How to Use Reward-Based Crowdfunding to Cocreate with Customers", 2018.
[24] Danmayr, F., Archetypes of crowdfunding platforms: A multidimensional comparison, Springer Science \& Business Media, 2013.

[25] Glass, R.L. and I. Vessey, "Contemporary application-domain taxonomies", IEEE Software, 12(4), 1995, pp. 63-76.

[26] McKnight, D.H., V. Choudhury, and C. Kacmar, "Developing and validating trust measures for ecommerce: An integrative typology", Information systems research, 13(3), 2002, pp. 334-359.

[27] Nickerson, R.C., U. Varshney, and J. Muntermann, "A method for taxonomy development and its application in information systems", European Journal of Information Systems, 22(3), 2013, pp. 336-359.

[28] Hevner, A. and S. Chatterjee, "Design science research in information systems", in Design research in information systems. 2010. Springer.

[29] Anderberg, M.R., Cluster analysis for applications, Office of the Assistant for Study Support Kirtland AFB N MEX, 1/1/1973.

[30] https://vitalik.ca/general/2017/06/09/sales.html, accessed 6-12-2018.

[31] Hausch, D.B., D.E. Logue, and J.K. Seward, "Dutch auction share repurchases: Theory and evidence", Journal of Applied Corporate Finance, 5(1), 1992, pp. 44-49.

[32] Boreiko, D. and N.K. Sahdev, "To ICO or not to ICO-Empirical analysis of Initial Coin Offerings and Token Sales", Social Science Research Network (SRRN), 2018, pp. 1-33.

[33] Hair, J.F., W.C. Black, B.J. Babin, R.E. Anderson, and R.L. Tatham, "Multivariate data analysis . Uppersaddle River", Multivariate Data Analysis (5th ed) Upper Saddle River, 1998.

[34] Everitt, B.S., S. Landau, M. Leese, and D. Stahl, "Hierarchical clustering", Cluster Analysis, 5th Edition, 2011, pp. 71-110.

[35] Fridgen, G., F. Regner, A. Schweizer, and N. Urbach, "Don't Slip on the Initial Coin Offering (ICO): A Taxonomy for a Blockchain-enabled Form of Crowdfunding", Twenty-Sixth European Conference on Information Systems, 2018, pp. 1-17. 